\title{
A Gradient Neural Network for online Solving the Time-varying Inverse Kinematics Problem of Four-wheel Mobile Robotic Arm
}

Zhou, Y

http://hdl.handle.net/10026.1/17287

10.1109/ddcls52934.2021.9455633

2021 IEEE 10th Data Driven Control and Learning Systems Conference (DDCLS) IEEE

All content in PEARL is protected by copyright law. Author manuscripts are made available in accordance with publisher policies. Please cite only the published version using the details provided on the item record or document. In the absence of an open licence (e.g. Creative Commons), permissions for further reuse of content should be sought from the publisher or author. 


\title{
A Gradient Neural Network for online Solving the Time-varying Inverse Kinematics Problem of Four-wheel Mobile Robotic Arm
}

\author{
Yanpeng Zhou ${ }^{1}$, Keping Liu ${ }^{1}$, Chunxu Li ${ }^{2}$, Gang Wang ${ }^{1}$, Yongbai Liu ${ }^{1}$, Zhongbo Sun*1 \\ 1. Department of Control Engineering, Changchun University of Technology, Changchun 130012, China \\ E-mail: liukeping@ccut.edu.cn, zhongbosun2012@163.com, yanpengzhou2020@163.com,.wg960109@163.com, \\ 2201804053@stu.ccut.edu.cn \\ 2. University of Plymouth, Centre for Robotics and Neural Systems, Plymouth PL48AA, UK \\ E-mail: chunxu.li@plymouth.ac.uk
}

\begin{abstract}
In this paper, a gradient neural network (GNN) is presented, analyzed and discussed to solve the time-varying inverse kinematics solution of the four-wheel mobile robotic arm, which can approximate the time varying inverse kinematics solution. A monolithic kinematics model of mobile robotic arm is established, and the inverse kinematics solution can synchronously coordinate the control of the mobile platform and the robotic arm to accomplish the task of the end-executor. Besides, the computer numerical results are provided to attest validity and high exactitude of GNN model in settling the time-varying inverse kinematics of a four-wheel mobile robotic arm.
\end{abstract}

Key Words: Mobile Robotic Arm, Time-varying Inverse Kinematics, Gradient Neural Network, Trajectory Tracking

\section{Introduction}

In recent decades, due to the expansion of the operating space of the robotic arm, and improving the ability of the mobile platform to interact with the environment, mobile robotic arm have aroused great interest in the fields of industry, medical treatment, service, geological survey, disaster rescue [1-3].

As one of the typical structures of mobile robots, the mobile robotic arm is constituted of a mobile platform and a robotic arm fastened on the mobile platform. In this way, the mobile robotic arm has the dual properties of the vastness of the working space of the mobile robot and the flexibility of the operating space of the robot [4-6]. According to the movement form, the mobile robotic arm can be divided into four types: wheeled mobile robotic arm, crawler mobile robotic arm, leg mobile robotic arm and hybrid mobile robotic arm. Among them, the wheeled robotic arm not only has a flexible structure, but also is easier to be controlled in practical applications. Owing to the different control objectives, the motion control of the mobile robotic arm is divided into two types: path planning and trajectory tracking [7]. Among them, the trajectory tracking control of the mobile robotic arm is a kind of time-varying inverse kinematics solution problem, which has been a hot and difficult problem.

Time-varying nonlinear problem is a significant branch of nonlinear problems, which exist widely in practical applications. In terms of control theory, since the wheeled mobile robotic arm is a highly coupled nonlinear system, it is very difficult to work out the trace tracking of wheeled mobile robotic arm. Due to the accuracy and flexibility of mobile robotic arm, more and more algorithms for the trajectory tracking of mobile robotic arm have been developed and verified, for example, nonlinear feedback control [8], sliding-mode control [9], robust control [10], adaptive control [11, 20, 21]. In [12], a holistic dynamic model of the mobile robotic arm is instituted directly, and then taking advan-

The work was supported by the Key Science and Technology Projects of Jilin Province, China, under Grant 20190302025GX. tage of nonlinear negative feedback to linearize and decouple the model, and finally the coordinated control of the mobile robotic arm is realized by an event-based control method. In [13], aiming at the trace tracking control problem of the wheeled mobile robotic arm, a radial basis function (RBF) neural network is employed to realize compensation of the unmodeled dynamics of system and external interference, and the control law is devised by the sliding mode control method. In [14], A robust adaptive controller is proposed to solve dynamic system problems, which has parametric and nonparametric uncertainties. It utilizes adaptive control technology to compensate parameter uncertainties, and suppresses bounded disturbances by sliding mode control.

For the various controllers mentioned above, it is necessary to establish a dynamic model of the system. Due to the highly coupled nonlinearity of the system, the holistic dynamic model of the mobile platform and the manipulator is very complicated. Therefore, It is very difficult to solve the time-varying inverse kinematics problem in real time, and the requirements for hardware/circuit implementation are relatively high. Relatively speaking, it is relatively simple to establish the overall kinematics model of the mobile manipulator, and its inverse kinematics solution can synchronize and coordinate the control of the mobile platform and the manipulator to complete the trajectory tracking task. In addition, the neural dynamics method has the characteristics of parallel distribution and easy hardware/circuit implementation, and is considered to be a powerful substitute for online matrix related problems. The traditional matrix inversion scheme is optimized based on gradient descent, which is essentially a constant matrix. Time-varying matrix problems are also solved by gradient neural networks. In [15], gradient neural networks based on global asymptotic convergence The network can solve non-singular matrix inversion problems online.

The article transforms the trace tracking problem of the mobile robotic arm into a time-varying matrix solution problem. In order to obtain the time-varying matrix solution, a gradient neural network is introduced to settle trace tracking 
problem. By integrating a robotic arm and a mobile platform into a system, a solution obtained by the equation designed by the GNN model simultaneously coordinate the mobile platform and a robotic arm for accomplishing the end effecter grasping task. Then, the simulation results ulteriorly prove the validity of the GNN model in settling the timevarying inverse kinematics of the mobile robotic arm [15].

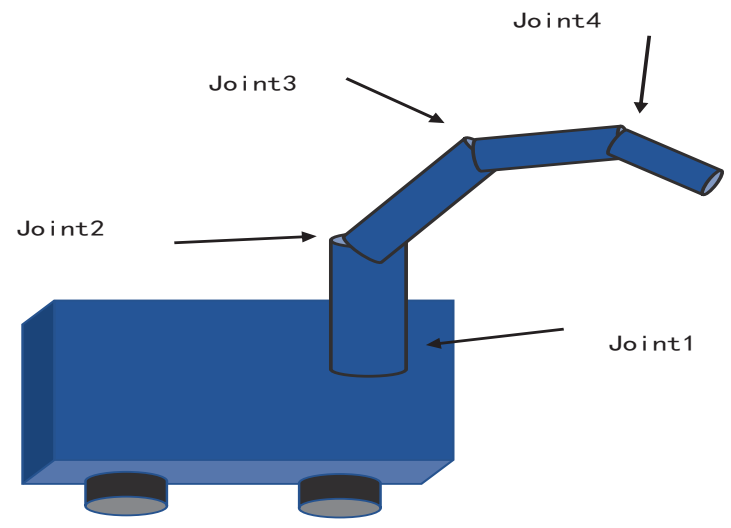

Fig. 1: Model of mobile robotic arm.

The rest of sections of this paper are structured into the following sections. Section 2 establishes the kinematical equation of mobile robotic arm, including the coordinate transformation equation of the robotic arm and the kinematical equation of the mobile platform. In Section 3, the gradient neural network with global exponential convergence and global stability is analyzed and studied for solving the trajectory tracking problem of the mobile robotic arm. In Section 4 , the mobile robotic arm controlled by the GNN model is presented by numerical simulations, and the results are analyzed to confirm the validity of the GNN model. Section 5 summarizes the full text and the outlook for future work. The primary tasks of this article are summed up as follows:

- Establishing a monolithic kinematical equation of a mobile platform and a robotic arm, and the solution to synergistically control the end effecter of the mobile robotic arm to accomplish the grasping task.

- In this paper, a GNN model is presented and analyzed for efficiently settling time-varying inverse kinematics problems.

- The experimental simulation demonstrates that the validity and veracity of the GNN model in the pathtracking of a wheeled mobile robotic arm.

\section{Kinematic Modeling of Mobile Robotic Arm}

This section not only directly provides the positive kinematics model of the robotic arm, but also analyzes and establishes the kinematics model of the mobile platform. Integrating the kinematical equation of the robotic arm and the kinematical equation of the mobile platform into a systematic equation, and finally receiving the kinematical equation of the entire mobile robotic arm. In addition, the mobile robotic arm is made up of a mobile platform and a fourdegree-of-freedom robotic arm, as shown in Figure 1, and top view of mobile platform is illustrated in Fig. 2 with related parameters summarized in Table 1 .

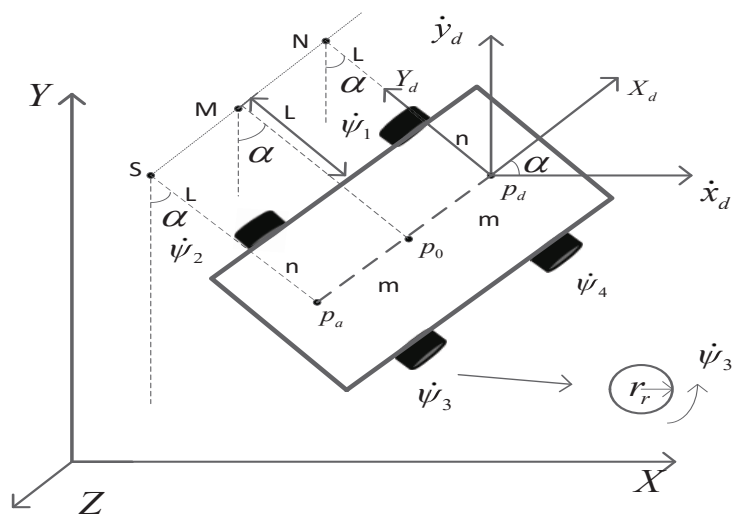

Fig. 2: Top view of mobile platform with related parameters.

\subsection{Kinematics of Four-joint Maniputor}

For a four degree-of-freedoms robotic arm, the end position of the mobile robotic arm in the world coordinate frame is generalized as below (i.e.,the transition of joint space vector $\varpi \in R^{n}$ to end effecter position and steering vector $r_{b} \in$ $\left.R^{m}\right)[16,17]$ :

$$
f(\varpi)=r_{b},
$$

where $f(\cdot)$ is a fluxionary nonlinear function of specified robotic arm with known structure and arguments. The coordinate transformation equation of the robotic arm is established as follows:

$$
f(\varpi)=\left[\begin{array}{c}
l_{1} c_{1} c_{2}+l_{2} c_{23} c_{1}+l_{4} c_{23} c_{1} c_{4}-l_{4} \mathrm{~s}_{23} c_{1} \mathrm{~s}_{4} \\
l_{1} \mathrm{~s}_{1} c_{2}+l_{2} c_{23} \mathrm{~s}_{1}+l_{4} c_{23} \mathrm{~s}_{1} \mathrm{c}_{4}-l_{4} \mathrm{~s}_{23} \mathrm{~s}_{1} \mathrm{~s}_{4} \\
l_{2} \mathrm{~s}_{23}+l_{1} \mathrm{~s}_{2}+l_{4} \mathrm{c}_{23} \mathrm{~s}_{4}+l_{4} \mathrm{~s}_{23} \mathrm{~s}_{4}+l_{3}
\end{array}\right] .
$$

where $\mathrm{c}_{j}:=\cos \varpi_{j}, \mathrm{~s}_{j}:=\sin \varpi_{j}, \mathrm{c}_{23}:=\cos \left(\varpi_{2}+\varpi_{3}\right)$ and $\mathrm{s}_{23}:=\sin \left(\varpi_{2}+\varpi_{3}\right)$ with $j=1,2, \ldots, 4, l_{1}=0.135, l_{2}=$ $0.147, l_{3}=0.103, l_{4}=0.035$.

\subsection{Kinematics of Mobile Platform}

According to the nonholonomic constraints of the mobile platform, a mathematical model is established in this subsection $[18,19]$. It should be pointed out that each part of the two subsystems of the mobile robotic arm is rigid, and the mobile platform only moves in the XOY plane. Moreover, for simplicity and clarity, lateral sliding is not considered, at the same time, the speed of four wheels is strictly vertical to the drive shaft. The Eq.(3) through Eq.(5) is derived from the relationship among the angular velocity, radius, velocity and rotation angle of the four wheels on the mobile platform. Owing to the above two points $p_{d}$ and $p_{0}$ of the same rigid body, the velocity projection theorem is satisfied, i.e., which the velocity projection of the two points on the $X_{d}$ axis is equal. Note that Eq.(4) indicates the velocity constraints in the horizontal direction of the mobile robotic arm (i.e., the mobile platform must satisfy the above relation.) Moreover, the kinematic constraint on the $Y_{d}$ axis is that the velocity in this direction is equivalent to zero, and it corresponds to the mobile platform without side sliding. Therefore, the kinematic equation of the mobile platform in the $Y_{d}$ axis is Eq.(3):

$$
\dot{x}_{d} \sin \alpha-\dot{y}_{d} \cos \alpha+m \dot{\alpha}=0
$$


Table 1: The parameters of the mobile platform

\begin{tabular}{|c|c|}
\hline Symbol & Description \\
\hline$P_{0}$ & The intersection of the diagonals of the monolithic mobile platform; The coordinates are $\left(x_{0}, y_{0}, z_{0}\right)$ \\
\hline$P_{d}$ & The connection point of mobile platform and 4-DOF robotic $\operatorname{arm}\left(X_{d}, Y_{d}, 0\right)$ \\
\hline $\mathrm{m}$ & The length of point $P_{0}$ and point $P_{d}$ (equivalent to $P_{0}$ to $P_{a}$ ) \\
\hline $\mathrm{n}$ & The space of the wheel 1 and $P_{d}$ point (equivalent to the space of the wheel 2 and $P_{a}$ point) \\
\hline $\mathrm{r}_{\mathrm{r}}$ & The radius of the four wheels \\
\hline$\alpha$ & Turning angle of mobile platform \\
\hline & Its time differential coefficient is the rotative velocity $\dot{\alpha}$ \\
\hline M & The mobile platform rotates around its imaginary point \\
\hline $\mathrm{N}$ & The imaginary point around which wheels 1 and 4 of the mobile platform rotates \\
\hline $\mathrm{S}$ & The imaginary point around which wheels 2 and 3 of the mobile platform rotates \\
\hline $\mathrm{L}$ & The space of point $\mathrm{N}$ and wheel 1 (equivalent to the distance between point $\mathrm{S}$ and wheel 2) \\
\hline & The rotational speed of a mobile platform around a point $\mathbf{M}$, and it is equal to $\omega_{r}=\dot{\alpha}$ \\
\hline$\dot{\psi}_{1} \dot{\psi}_{2} \dot{\psi}_{3} \dot{\psi}_{4}$ & The velocity of wheel 1 , wheel 2 , wheel 3 and wheel 4 \\
\hline
\end{tabular}

$$
\begin{gathered}
\dot{\alpha}=\frac{\mathrm{r}_{\mathrm{r}} \dot{\psi}_{1}}{L}=\frac{\dot{x}_{0} \cos \alpha+\dot{y}_{0} \sin \alpha}{L+\mathrm{n}}=\frac{\mathrm{r}_{\mathrm{r}} \dot{\psi}_{4}}{L+2 \mathrm{n}}, \\
\dot{x}_{0} \cos \alpha+\dot{y}_{0} \sin \alpha=\dot{x}_{d} \cos \alpha+\dot{y}_{d} \sin \alpha,
\end{gathered}
$$

Due to Eq.(3) and Eq.(4), the equation of the angular velocity of the mobile robotic arm and the angular velocity of the four wheels of the mobile platform can be calculated, and the equation between the velocity of the mobile platform $\dot{x}_{d}$ $\dot{y}_{d}$ and the four wheels $\psi_{1}, \psi_{2}, \psi_{3}, \psi_{4}$ are presented as:

$$
\dot{\alpha}=\frac{\mathrm{r}_{\mathrm{r}}}{4 \mathrm{n}}\left(\dot{\psi}_{4}-\dot{\psi}_{1}+\dot{\psi}_{3}-\dot{\psi}_{2}\right),
$$

$$
\begin{aligned}
& \dot{x}_{d}=\left(\frac{3 \mathrm{r}_{\mathrm{r}}}{4} \cos \alpha+\frac{\mathrm{mr}_{\mathrm{r}}}{4 \mathrm{n}} \sin \alpha\right) \dot{\psi}_{1}+\left(\frac{\mathrm{mr}_{\mathrm{r}}}{4 \mathrm{n}} \sin \alpha-\frac{\mathrm{r}_{\mathrm{r}}}{4} \cos \alpha\right) \dot{\psi}_{2} \\
& +\left(\frac{\mathrm{r}_{\mathrm{r}}}{4} \cos \alpha-\frac{\mathrm{mr}_{\mathrm{r}}}{4 \mathrm{n}} \sin \alpha\right) \dot{\psi}_{3}+\left(\frac{\mathrm{r}_{\mathrm{r}}}{4} \cos \alpha-\frac{\mathrm{mr_{ \textrm {r } }}}{4 \mathrm{n}} \sin \alpha\right) \dot{\psi}_{4}, \\
& \dot{y}_{d}=\left(\frac{3 \mathrm{r}_{\mathrm{r}}}{4} \sin \alpha-\frac{\mathrm{mr}_{\mathrm{r}}}{4 \mathrm{n}} \cos \alpha\right) \dot{\psi}_{1}-\left(\frac{\mathrm{r}_{\mathrm{r}}}{4} \sin \alpha+\frac{\mathrm{mr}_{\mathrm{r}}}{4 \mathrm{n}} \cos \alpha\right) \dot{\psi}_{2} \\
& +\left(\frac{\mathrm{r}_{\mathrm{r}}}{4} \sin \alpha+\frac{\mathrm{mr}_{\mathrm{r}}}{4 \mathrm{n}} \cos \alpha\right) \dot{\psi}_{3}+\left(\frac{\mathrm{r}_{\mathrm{r}}}{4} \sin \alpha+\frac{\mathrm{mr}_{\mathrm{r}}}{4 \mathrm{n}} \cos \alpha\right) \dot{\psi}_{4},
\end{aligned}
$$

For the Eq.(7), the two variables $\dot{x_{d}}$ and $\dot{y_{d}}$ are the derivatives of $x_{d}$ and $y_{d}$ with respect to time $t$, the kinematical equation is simplified as follows:

$$
\begin{aligned}
& \mathrm{D} \dot{\psi}=\dot{\alpha} \\
& \mathrm{C} \dot{\psi}=\dot{p}_{d}
\end{aligned}
$$

where

$$
\begin{gathered}
\dot{\psi}=\left[\dot{\psi}_{1}, \dot{\psi}_{2}, \dot{\psi}_{3}, \dot{\psi}_{4}\right]^{\mathrm{T}}, \\
\dot{p}_{d}=\left[\dot{x}_{d}, \dot{y}_{d}\right]^{\mathrm{T}},
\end{gathered}
$$

The matrixes D and $\mathrm{C}$ are defined as follows:

$$
\begin{gathered}
\mathrm{D}=\frac{\mathrm{r}_{\mathrm{r}}}{4 \mathrm{n}}[-1,-1,1,1] \\
\mathrm{C}=\frac{\mathrm{r}_{\mathrm{r}}}{4}\left[\begin{array}{cc}
\cos \alpha & -\sin \alpha \\
\sin \alpha & \cos \alpha
\end{array}\right]\left[\begin{array}{cccc}
3 & -1 & 1 & 1 \\
\frac{-\mathrm{m}}{\mathrm{n}} & \frac{-\mathrm{m}}{\mathrm{n}} & \frac{\mathrm{m}}{\mathrm{n}} & \frac{\mathrm{m}}{\mathrm{n}}
\end{array}\right] .
\end{gathered}
$$

Assuming that $\mathrm{r}_{\mathrm{r}}=0.052 \mathrm{~m}, \mathrm{n}=0.3 \mathrm{~m}$ and $\mathrm{m}=0.1 \mathrm{~m}$.

\subsection{The Global Kinematic Equation of the Mobile Robotic Arm}

Combining Eq.(2) with Eq.(7), the global kinematical equation of the mobile robotic arm can be obtained with kinematical equation of mobile platform and kinematical equation of the robotic arm. By employing the transformational matrix, the kinematical equation is transformed from the base coordinate system to the world coordinate frame with the position equation of the end-effecter of the robotic arm is also concerned with the world coordinate frame.

The global kinematical equation of the mobile robotic arm is the kinematical equation of the end position of the robotic arm in the world coordinate frame.

$$
\begin{aligned}
& z_{d}=\left[\begin{array}{cccc}
\cos \alpha & -\sin \alpha & 0 & x_{d} \\
\sin \alpha & \cos \alpha & 0 & y_{d} \\
0 & 0 & 1 & 0
\end{array}\right]\left[\begin{array}{c}
f(\varpi) \\
1
\end{array}\right] \\
& z_{d}:=\left[\begin{array}{c}
x_{d} \\
y_{d} \\
0
\end{array}\right]+h(\varpi, \alpha) .
\end{aligned}
$$

By differentiating the above formula in relation to time $t$, the kinematical equation of the velocity level of the mobile robotic arm in the world coordinate frame is received as follows:

$$
\dot{z}_{d}=\left[\begin{array}{c}
\dot{x}_{d} \\
\dot{y}_{d} \\
0
\end{array}\right]+J(\alpha, \varpi)\left[\begin{array}{c}
\dot{\alpha} \\
\dot{\varpi}
\end{array}\right]
$$

where jacobian matrix $J(\alpha, \varpi)$ is defined as $J(\alpha, \varpi)=$ $\partial h(\alpha, \varpi) / \partial v$ and $v=\left[\varpi^{\mathrm{T}}, \alpha\right]^{\mathrm{T}}$. Eliminating unimportant variables by adopting (8), furthermore, the simplified equation of the mobile robotic arm is acquired as:

$$
\dot{z}_{d}=\left[\begin{array}{c}
\mathrm{C} \dot{\psi} \\
0
\end{array}\right]+J(\alpha, \varpi)\left[\begin{array}{c}
\mathrm{D} \dot{\psi} \\
\dot{\varpi}
\end{array}\right] .
$$

where $q=\left[\psi, \varpi^{\mathrm{T}}\right]^{\mathrm{T}}$ (i.e., the combined angle vector), represents the angle vector of the mobile robotic arm, which involve the wheel angle of rotation of the mobile platform and the rotation angle of each joint of the robotic arm. Eq.(15) can be further reduced to a compact matrix as:

$\dot{z}_{d}=\left[\begin{array}{cc}\mathrm{C} & 0 \\ 0 & 0\end{array}\right]\left[\begin{array}{c}\dot{\psi} \\ \dot{\varpi}\end{array}\right]+J(\alpha, \varpi)\left[\begin{array}{cc}\mathrm{D} & 0 \\ 0 & I\end{array}\right]\left[\begin{array}{c}\dot{\psi} \\ \dot{\varpi}\end{array}\right]:=G \dot{q}$,

where $I$ is an identity matrix, a coefficient matrix $G$ is defined as below:

$$
G=\left[\begin{array}{cc}
\mathrm{C} & 0 \\
0 & 0
\end{array}\right]+J(\alpha, \varpi)\left[\begin{array}{cc}
\mathrm{D} & 0 \\
0 & I
\end{array}\right] .
$$

Kinematics equation (16) of the velocity level of the mobile robotic arm corresponding to the world coordinate 
frame. To facilitate writing, the global kinematical equation (13) of the mobile robotic arm is transformed into a simplified form:

$$
z_{d}(t)=\delta(q, t)
$$

\section{Gradient Neural Network}

It is deserved to point out that, in practical industrial applications, the kinematics equation (13) at the position level is aimed at a time-varying system. Therefore, it is very significant to resolve the problem of time-varying system with relatively accurate solutions. A gradient neural network is presented to resolve inverse kinematics problem of time-varying system for mobile robotic arm [22-25, 27].

First, the kind of neural network is applied to resolve inverse of nonsingular constant matrices. The definition of the inverse of a matrix can be put forward and as follows:

$$
A X-I=0 .
$$

where $I$ represents an identity matrix, and signifies an unknown matrix to be solved, for the inverse of the matrix $A^{-1}$.

Second, the method of dynamic system is employed to solve $X(t)$, which needs to design a norm-based error function of scalar value $\epsilon=\|A X(t)-I\|_{F}^{2} / 2$. When the error function is equal to $0, X(t)$ is an exact solution of Eq.(18). In the same way, the kinematics solution problem of the mobile robotic arm can also be transformed into a similar problem. The energy function of scalar value based on norm is $\epsilon=\left\|z_{d}(t)-\delta(q, t)\right\|_{2}^{2}$. When $\epsilon=0$, the minimum point of $\epsilon$ is obtained.

Third, a numerical procedure can be devised to develop into the decline orientation of the energy function $\epsilon$, up to getting the minimum $q$. Generally speaking, the decline orientation is the subtractive gradient of $\epsilon$, i.e., $-\partial \epsilon / \partial q$. According to the above processes that the differential coefficient of $\epsilon$ in regard to $q$ is derived as $-G^{\mathrm{T}}\left(z_{d}(t)-\delta(q, t)\right)$. Owing design formula $\dot{q}(t)=-\gamma \partial \epsilon / \partial q$, the dynamic equation of conventional gradient neural network is developed to settle the time-varying inverse kinematics of the mobile robotic arm, which is presented as follows:

$$
\dot{q}(t)=\gamma G^{\mathrm{T}}\left(z_{d}(t)-\delta(q, t)\right)
$$

where the argument $\gamma>0$, an inductance argument or the count backward of a capacitance argument, which can be set to the maximum allowed by the hardware, and be chosen properly when conducting experiments or simulations.

\section{Verification of GNN Model}

In this scetion, the effectiveness of the gradient neural network model(19) to settle the time-varying reverse kinematics of the mobile robotic arm is verified by simulation. Within reasonable range, setting a desired trajectory and the endeffecter of the robotic arm is expected to follow the trajectory. Generally, the incipient status of the variables is $\varpi(0)=[0,0,0,0, \pi / 12, \pi / 12, \pi / 12, \pi / 6]^{\mathrm{T}} \mathrm{rad}, \alpha(0)=$ $x_{d}(0)=y_{d}(0)=0$ and $\gamma=100$ [26].

In the subsection, the end position of the mobile robotic arm is anticipated tracking a stated trajectory, the simulation time was $70 \mathrm{~s}$, and the simulation result is reported and presented as follows:

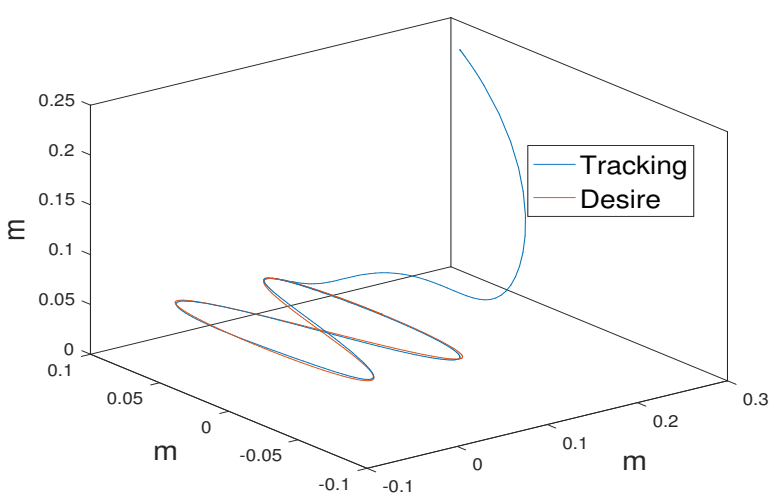

Fig. 3: The desired trajectory and actual trajectory of the mobile robotic arm.

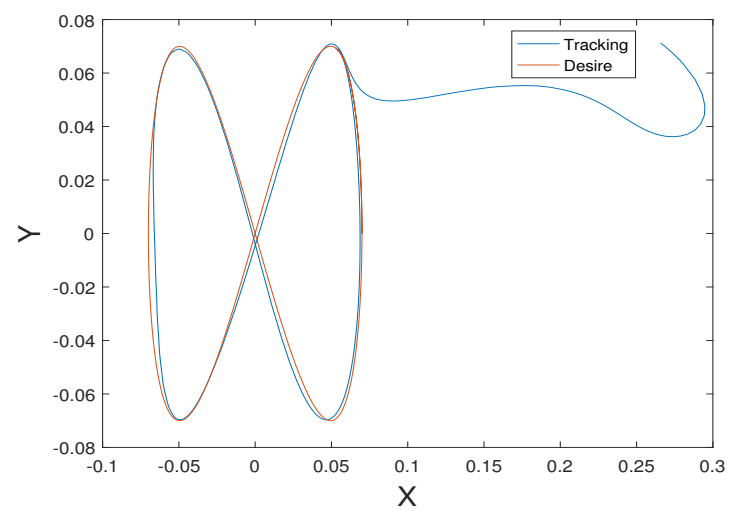

Fig. 4: Top view of the desired trajectory and actual trajectory of the mobile robotic arm.

Fig. 3 shows the end-effector trajectory is super imposed over the desired path, which the completion degree of the track task is indicated, which reflects the GNN controller can obtain a favourable control result. Fig. 4 is a top view of Fig. 3. It is perceived from Fig. 4 that the end motion trajectory of the mobile robotic arm is awfully close to the stated path.

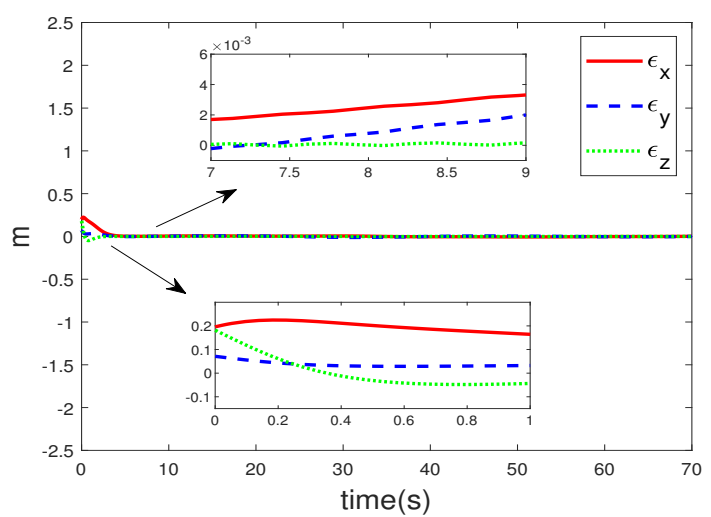

Fig. 5: Position error synthesized by GNN model in the mobile robotic arm path-tracking.

Fig. 5 shows position error $\left(\epsilon=\left[\epsilon_{x}, \epsilon_{y}, \epsilon_{z}\right]^{\mathrm{T}}:=z_{d}-\right.$ $\left.h(\alpha, \varpi)-\left[x_{d}, y_{d}, 0\right]^{\mathrm{T}}\right)$ of the mobile robotic arm end effecter tracking the desired trajectory. It can be surveyed from the Fig. 5 that the incipient error is large, because the incip- 
ient state of the mobile robotic arm is unreasonable. After a period of time, the trajectory of the end effecter of the robotic arm synchronizes with the stated trajectory, which can be seen from the simulation image that the tracking error is less than $4 \times 10^{-3} \mathrm{~m}$.

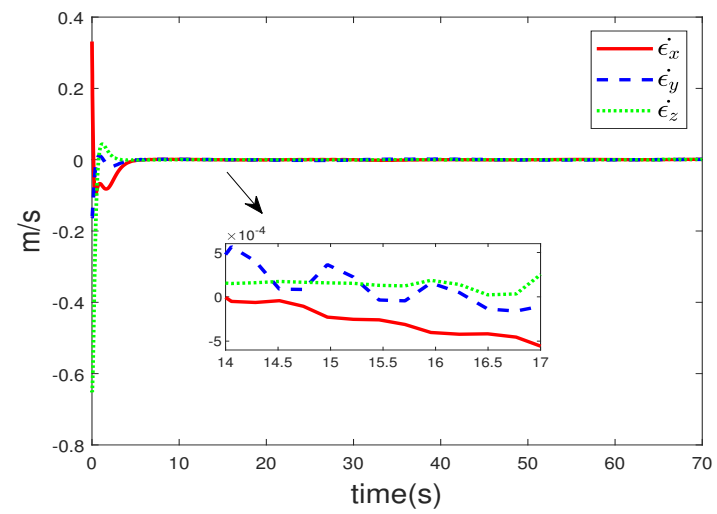

Fig. 6: Velocity error synthesized by GNN model in the mobile robotic arm path-tracking.

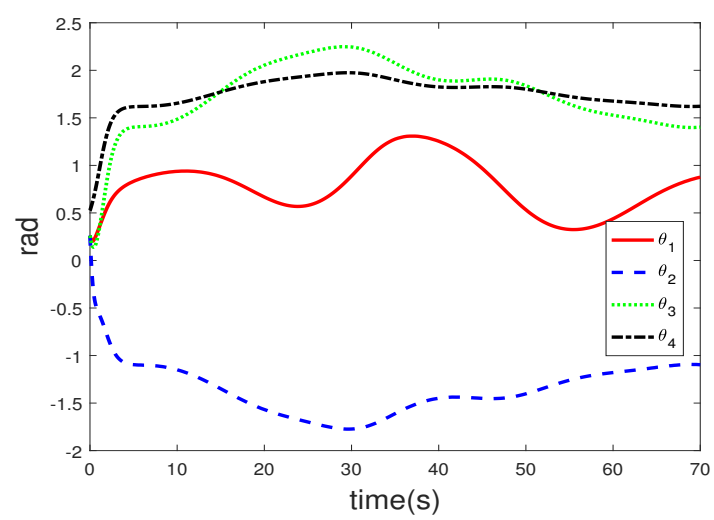

Fig. 7: Joint angle of robotic arm synthesized by GNN model in path-tracking.

Fig. 6 demonstrates the variation of tracking velocity error of the end effecter of the robotic arm. The convergence speed approaches 0 in a short time, reflecting the speedability effect of the GNN controller. The variation of velocity error in the later period is almost 0 , which reflects the stability of the controller. It is surveyed from Fig. 6, the variation of tracking velocity errors in the $\mathrm{x}$-axis, $\mathrm{y}$-axis, and $\mathrm{z}$-axis, which are also acceptable in practice. The simulation results show that the mobile robotic arm synthesized by the GNN model (19) can commendably complete the trajectory tracking task.

In addition, considering the actual industrial conditions, it is generally necessary to concern the situation variations of other parameters of the mobile robotic arm, for example, the angle variation of each wheel of the mobile platform. The mobility and stability of the mobile platform are extremely important in terms of application. When the mobile platform runs smoothly, it will not conflict with joint motion and reduce the impact on the position of the end robotic arm. Fig. 7 to Fig. 10 show the variation of the joint angle of the robotic arm, variation in angular velocity of the joint, the rotation angle of the wheels of the mobile platform, and the rotational velocity of the wheels when the mobile robotic arm tracks the desired trajectory.

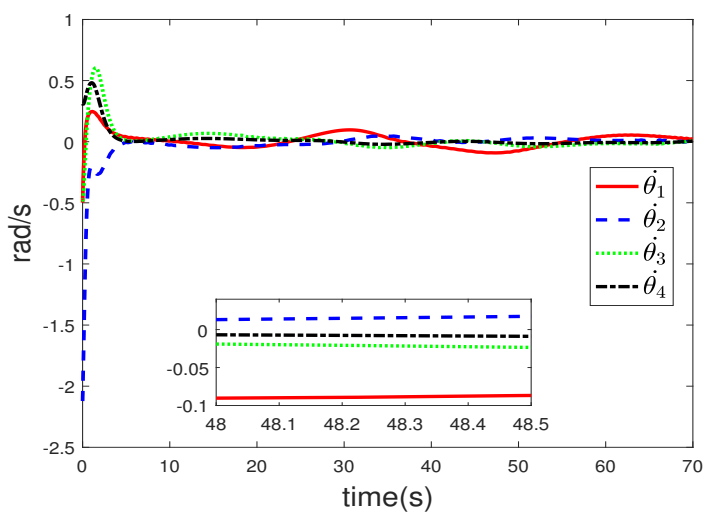

Fig. 8: Joint angle velocity of robotic arm synthesized by GNN model in path-tracking.

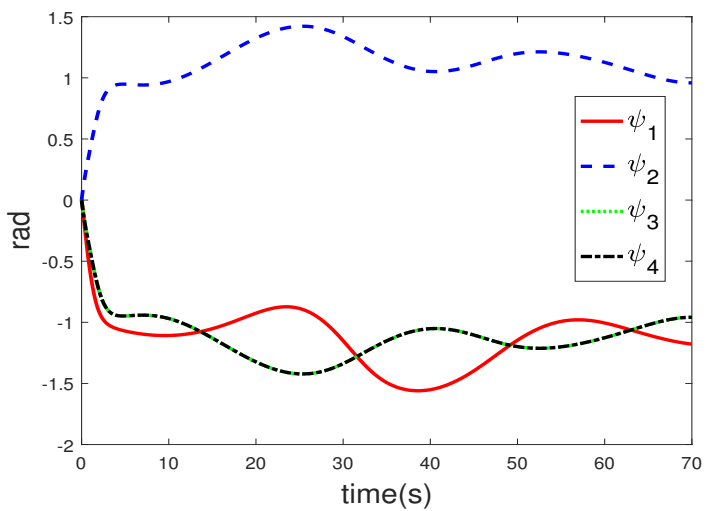

Fig. 9: Angle of mobile platform wheels synthesized by GNN model in path-tracking.

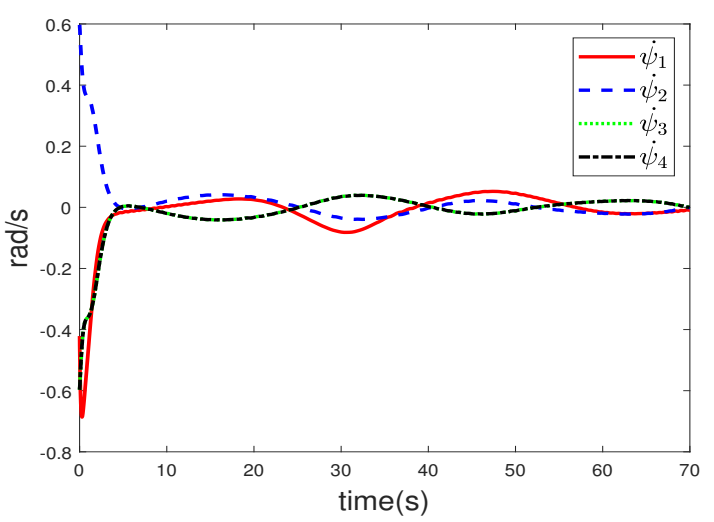

Fig. 10: Angular velocity of mobile platform wheels synthesized by GNN model in path-tracking.

The solutions of the time-varying reverse kinematics problem of the mobile robotic arm position level are shown in Fig. 7, Fig. 8, Fig. 9, Fig. 10. It can be demonstrated that 
the mobile platform and the robotic arm always maintain coordinated movement to complete the grasping task of the end effecter.

Fig. 7 and Fig. 8 depict the variations in the angle and angular velocity of each joint of the robotic arm. Fig. 9 and Fig. 10 show the variations in the angle and velocity of the wheels of the mobile platform. The final velocity of angular velocity and velocity of the wheels are equal to zero, which proves that the end effecter of the mobile robotic arm stops moving after completing the task. And each variable variations steadily over time, there is no sudden variation, hence, the control method is feasible in practice. Therefore, utilizing the GNN control model to solve the time varying inverse kinematics of the mobile robotic arm can realize the purpose of accurate control.

\section{Conclusion}

In this paper, aiming at the trajectory tracking control of the mobile robotic arm, a GNN model is proposed and studied to settle this problem. The GNN model can be used to acquire an pinpoint solution of time-varying inverse kinematics. The effectiveness of the GNN model controller can be surveyed through simulation results, and the model can effectively achieve high-precision trajectory tracking control. Some areas for improvement in the future: a peculiar type of recurrent neural network, named zeroing neural network, will be investigated, which can quickly and precisely solve to the solution of the time-varying inverse kinematics of the mobile robotic arm. Besides, a noise-tolerant neural network will be considered to deal with noises.

\section{References}

[1] L. Jin, S. Li. Distributed task allocation of multiple robots: A control perspective[J]. IEEE Transactions on Systems, Man, and Cybernetics: Systems,693-701,2018.

[2] L. Jin, S. Li, H. La, X. Luo. Manipulability optimization of redundant manipulators using dynamic neural networks[J]. IEEE Transactions on Industrial Electronics,4710-4720,2017.

[3] D. Belanche, J. Schepers , E. Bridges, et al. Service Robot Implementation: a Theoretical Framework and Research Agenda[J]. Service Industries Journal,203-225,2020.

[4] R. Ram, P. Pathak, S. Junco. Inverse Kinematics of Mobile Manipulator Using Bidirectional Particle Swarm Optimization by Manipulator Decoupling[J]. Mechanism and Machine Theory, 131:385-405,2018.

[5] Y. Wei, W. Jiang, A. Rahmani, et al. Motion Planning for a Humanoid Mobile Manipulator System[J]. International Journal of Humanoid Robotics, 16(2):1950006.1-1950006.31,2019.

[6] J. Z. Zhang, L. Jin, L. Cheng. RNN for perturbed manipulability optimization of manipulators based on a distributed scheme: A game-theoretic perspective[J].IEEE Transactions on Neural Networks and Learning Systems,31:5116-5126,2020.

[7] S. Mishra, P. Londhe, S. Mohan, et al. Robust Task-space Motion Control of a Mobile Manipulator Using a Nonlinear Control with an Uncertainty Estimator[J]. Computers \& Electrical Engineering,297-302,2017.

[8] J. Wu, Q. Chen, et al. Adaptive Neural Dynamic Surface Control with Prespecified Tracking Accuracy of Uncertain Stochastic Nonstrict-Feedback Systems[J]. IEEE Transactions on Cybernetics, 99:1-14,2020.

[9] A. Jebri, T. Madani, K. Djouani, et al. Robust Adaptive Neuronal Controller for Exoskeletons with Sliding-Mode[J]. Neurocomputing,317-330,2020.
[10] J. Zhao, J. Na, G. Gao. Adaptive Dynamic Programming Based Robust Control of Nonlinear Systems with Unmatched Uncertainties[J]. Neurocomputing,56-65,2020.

[11] J. Shi, D. Yue, X. Xie. Adaptive Optimal Tracking Control for Nonlinear Continuous-time Systems with Time Delay Using Value Iteration Algorithm[J]. Neurocomputing, 172-178,2019.

[12] J. Tan, N. Xi. Integrated Task Planning and Control for Mobile Manipulators [J].International Journal of Robotics Research,22(5):337-356,2003.

[13] W. Dong, W. Xu. Adaptive Tracking Control of Uncertain Nonholonomic Dynamic System[J].IEEE Trans Autom Control,46(3):450-454,2001.

[14] Z. Li, Y. Yang, J. Li. Adaptive Motion/Force Control of Mobile Under-actuated Manipulators with Dynamics Uncertainties by Dynamic Coupling and Output Feedback[J].IEEE Trans Control Syst Technol,5(18):1068-1079,2010.

[15] Y. Zhang, Y. Shi, K. Chen, et al. Global Exponential Convergence and Stability of Gradient-based Neural Network for Online Matrix Inversion[J].Applied Mathematics \& Computation, 215(3):1301-1306,2009.

[16] J. Moreno-Valenzuela, L. Gonzlez-Hernndez. Operational Space Trajectory Tracking Control of Robot Manipulators Endowed with a Primary Controller of Synthetic Joint Velocity[J]. ISA transactions,50(1):131-140,2011.

[17] Y. Zhang, S. Li, et al. Recurrent Neural Network for Kinematic Control of Redundant Manipulators with Periodic Input Disturbance and Physical Constraints[J]. IEEE Transactions on Cybernetics,4194-4205,2018.

[18] S. Li, Y. Zhang, L. Jin. Kinematic Control of Redundant Manipulators Using Neural Networks[J]. IEEE Transactions on Neural Networks and Learning Systems,2243-2254,2017.

[19] L. Tan. Distributed Optimal Control for Nonholonomic Systems with Input Constraints and Uncertain Interconnections[J]. Nonlinear Dynamics,801-817,2018.

[20] W. Qinglai, L. Derong. Value Iteration Adaptive Dynamic Programming for Optimal Control of Discrete-Time Nonlinear Systems[J].IEEE Transactions on Cybernetics,840-853,2016.

[21] N. Tan Luy. Robust adaptive dynamic programming based online tracking control algorithm for real wheeled mobile robot with omni-directional vision system[J]. Transactions of the Institute of Measurement \& Control,832-847,2016.

[22] Z. T. Xie, L. Jin. RNN for repetitive motion generation of redundant robot manipulators: An orthogonal projection-based scheme[J].EEE Transactions on Neural Networks and Learning Systems, 1-14,2020.

[23] Z. Tan, Y. Hu, K. Chen. On the Investigation of Activation Functions in Gradient Neural Network for Online Solving Linear Matrix Equation[J]. Neurocomputing,185-192,2020.

[24] X. Lv, Z. Tan, K. Chen, et al. Improved Recurrent Neural Networks for Online Solution of Moore-Penrose Inverse Applied to Redundant Manipulator kinematic Control[J]. Asian Journal of Control,1188-1196,2020.

[25] S. Park, M. Lee, J. Kim. Trajectory Planning with Collision Avoidance for Redundant Robots Using Jacobian and Artificial Potential Field-based Real-time Inverse Kinematics[J]. International Journal of Control, Automation and Systems,18(8):2095-2107,2020.

[26] L. Xiao, Y. Zhang. Solving time-varying inverse kinematics problem of wheeled mobile manipulators using Zhang neural network with exponential convergence[J]. Nonlinear Dynamics, 76(2):1543-1559,2014.

[27] L. Jin, J. k. Yan, X. j. Du, X. C Xiao. RNN for solving time-variant generalized Sylvester equation with applications to robots and acoustic source localization[J]. IEEE Transactions on Industrial Informatics,16(210):6359-6369,2020. 